\title{
APPLICATION OF SYNCHROTRON X-RAY TOPOGRAPHY TO THE STUDY OF MATERIALS
}

\author{
B.K. TANNER \\ Department of Physics, University of Durham \\ South Road, Durham, DH1 3LE, Great Britain
}

\begin{abstract}
In this article, we review the application of synchrotron X-ray topography to the study of the defect structure of materials. Following a discussion of source and detector requirements, in situ dynamic, stroboscopic, and ultra-high strain sensitivity experiments are discussed in the context of the new, third generation, synchrotron radiation sources. The intensity and continuous spectrum of synchrotron radiation is particularly important but further use of the time structure and polarization is timely. The future potential of the technique is discussed in the context of recent results at the European Synchrotron Radiation Facility in Grenoble.
\end{abstract}

PACS numbers: 07.85.+n, 61.10.Lx

\section{Introduction}

Since the work of Lang [1] in the late 1950's X-ray topography has gradually become accepted as a standard characterization technique for highly perfect crystals $[2,3]$. However, when performed with conventional generators the technique acquired a reputation for being slow and applicable only to very highly perfect crystals. In 1974 Tuomi et al. [1] showed that synchrotron radiation could be used to record multiple X-ray topographs in extremely short exposure times with a very simple experimental configuration. IIart [5] soon after demonstrated high resolution imaging with synchrotron radiation and this led to a major revolution in the type of experiment which could be performed and the nature of the data collected. In the intervening two decades steady developments have occurred, which have been recently reviewed in depth by Bowen and Tanner [6].

\section{X-ray topography}

$\mathrm{X}$-ray diffraction topography is the X-ray analogue of transmission electron microscopy in that the variation of intensity in one diffracted beam is recorded as a function of position. This is conventionally done photographically, although there is strong pressure to move to direct-recording electronic imaging devices. In the 
vicinity of local strains, there is change in scattering power and a pseudo-image is formed which provides a map of the strain variation across the crystal. X-ray topography is, in effect, the $\mathrm{X}$-ray analogue of conventional transmission electron microscopy. The simplest $\mathrm{X}$-ray topography experiment is to use the white synchrotron radiation beam to perform Laue topography, either in transmission or reflection. Double axis experiments [7], where one or more Bragg reflections are used to condition the beam in wavelength or divergence, enable smaller and long range strains to be detected. Triple axis experiments, where one or more reflections are used to analyse the X-rays scattered from the sample, enable tilts and dilations to be distinguished $[8,9]$. All three types of topography experiment have been performed at synchrotron radiation sources, although very few triple axis experiments have so far been reported.

\section{Source requirements for synchrotron X-ray topography}

In the laboratory, X-ray topography (XRT) is usually performed with characteristic radiation and it is straightforward to show that the exposure time for a section topograph scales with $P S_{i x}^{-2}$ and for a traverse topograph as $P S_{x}^{-1}$, where $P$ is the X-ray tube power and $S_{x}$ is the source dimension in the incidence plane [2]. The continuous nature of the synchrotron spectrum, however, results in a very different dependence on the source power and dimensions. From a simple consideration of the duMond diagram, Miltat [10] derived an expression for the intensity integrated normal to the incidence plane in a synchrotron radiation Laue topograph. However, exposure time is inversely proportional simply to the intensity (diffracted X-ray power per unit area) when a single absorbed X-ray photon results in one developable grain or pixel event. The intensity $I_{h}$ is given by

$$
I_{h}=A|C| \lambda^{3} P(\lambda) F_{h} b^{1 / 2} S_{x} / D^{2} \sin ^{2} O_{\mathrm{B}},
$$

where $b=\gamma_{0} /\left|\gamma_{h}\right|, C=1$ or $\cos 20_{\mathrm{B}}, F_{h}$ is the structure factor, $A$ is a constant and $O_{\mathrm{B}}$ is the Bragg angle. $D$ is the specimen to source distance, $P(\lambda)$ is the source power per unit wavelength interval, $\gamma_{0}=K_{0} \cdot n$ and $\gamma_{h}=K_{h} \cdot n$, where $n$ is a unit vector parallel to the inward normal to the specimen surface. $\boldsymbol{K}_{\boldsymbol{h}}$ and $\boldsymbol{K}_{0}$ are the diffracted and incident wave vectors.

While the first reaction to Eq. (1) is concluded that increasing the source brilliance is a disadvantage for $\mathrm{X}$-ray topographic experiments, closer examination shows this not to be the case. For all practical cases $m_{0} c^{2} / E D>S$, where $m_{0}$ is the electron rest mass, $E$ the electron energy and $S$ the source dimension. Then the resolution $R$ in a topograph is given by approximately

$$
R=d S / D
$$

where $d$ is the source to detector distance. The minimum value of $R$ is determined by the physical processes occurring within the detectors available, while $d$ is limited by the requirement to separate diffracted and forward transmitted beams. Combining Eqs. (1) and (2), we see that the intensity scales as $P / S_{x}$ as in a conventional laboratory topograph.

Ilowever, it must be emplasised that this intensity gain is only achieved by locating the experiment at the optimum distance $D$ from the source, which 
will decrease with decreased source size. For fixed $D$, the gain from increased brilliance is better resolution. As asymmetric Bragg reflections can be used to magnify X-ray images [11], detector resolution limitations can be overcome. Recent simulations of dislocation images in the Bragg case double crystal setting have shown that there exists significant contrast at submicron resolution [12] which is presently lost through geometrical (source size) and detector limitations. Much is to be gained from two topography stations on one beam line, one optimised for minimum exposure time, the other designed for ultra-high (submicron) resolution experiments.

Multipole wigglers clearly have major advantages in the enhanced power available for topographic experiments but undulators do not look promising. The emission from these devices is in an extremely narrow cone which, even at $100 \mathrm{~m}$, only corresponds to a topographic image area of a few $\mathrm{mm}^{2}$. This arises because the wavelength varies strongly over the cone angle and the different parts of the crystal cannot satisfy the Bragg condition simultaneously. To overcome this problem, the undulator must be detuned to such an extent that its performance is not significantly better than that of a multipole wiggler, but much more restricting in wavelength band. The advantage of limiting the unwanted power loading on the crystal can be circumvented in other ways, for example by use of filters. The SPring8 project team in Japan is presently undertaking experiments to assess the feasibility of using a grazing incidence transmission mirror to reflect the unwanted low energy radiation out of the beam. Some topographic experiments which exploit circular polarization are possible on undulator stations, but (presently at the European Synchrotron Radiation Facility (ESRF) do not constitute a sufficiently large class for a dedicated station.

\section{Detector's}

Without doubt, the major limitation in the exploitation of X-ray topography to dynamic, in silu experiments is in the performance of current detectors. The performance of a quantum limited detector is governed by the Rose-deVries law

$$
C^{2} \delta^{2} I \tau \xi / k^{2}=1,
$$

where $C$ is the contrast of the defect, $\delta$ is the resolution or pixel size, $I$ is the intensity, $\tau$ is the exposure time, $\xi$ is the detector quantum detection efficiency and $k$ is the system signal-to-noise ratio. Calculations of the performance of detectors with resolution of the order of 5 to 10 microns show that real time detection of defects with about $10 \%$ contrast should be feasible at sources such as the ESRF. While improvements in spatial resolution are clearly desirable, it is economically unrealistic to do other than exploit technology developed for alternative commercial purposes.

\section{Dynamic experiments}

White radiation topography has the following special features:

(i) Short exposures (from milliseconds up to minutes, depending on the source and the specimen); 
(ii) The ability to record multiple reflections on one plate;

(iii) The ability to image defects in crystals which contain long range elastic strains as each point on the specimen selects its own appropriate wavelength band for diffraction;

(iv) The ability to obtain overall strain maps of specimens that are too distorted for individual defects to be distinguished;

(v) A simple experimental arrangement that is well suited for experimental stages for in silu control of experimental variables.

This technique has therefore been much exploited for dynamic experiments. Of particular note are studies on the plastic deformation in ice which began in Japan, were extended by Whitworth and colleagues at Daresbury and most recently been continued by Dudley's group at Brookhaven. In situ straining and real-time topography were employed to follow the evolution of dislocation sources and measure velocity-stress relationships. Whilst the classic Frank-Read sources were occasionally observed, and emissary dislocations from inclusions or precipitates were seen as a source of dislocations, complex multiplication processes were observed that are still not understood [13].

Studies of the plastic deformation processes in ice are of very considerable interest as they help to shed light on the strange behaviour of glacier formation and propagation. The influence of slip on non-basal planes in ice was found to be unexpectedly high, and on these planes a very large ratio of edge to screw dislocation velocity was seen [14-16]. Image forces near crystal surfaces also played an important role in dislocation multiplication. In the study of the nucleation and propagation of dislocations from scratclics in a geomctry designed to produce slip on non-basal planes, edge dislocations were found to glide on non-basal planes, but screw dislocations were completely immobile except in the basal plane [15].

Shearwood and Whitworth [17] and IIondolı et al. [18] have completed very detailed measurements of the dislocation velocities using a stress pulse technique. Dislocations were found to glide on the basal plane as straight segments in both screw and $60^{\circ}$ orientation with velocity proportional to stress. The formation of straight glide segments in the basal plane is evidence that dislocations glide by propagation of kinks across a Pcierls barrier. Ilowever, activation energies determined for the two types show marked order of magnitude disagreement with current theoretical models. The activation energy data for non-basal dislocations suggests that proton disorder may limit dislocation mobility, though there is still a disagreement between the predicted and observed magnitudes for the dislocation velocities. Real-time synchrotron XRT has been used to measure self-diffusion rates in ice at the Photon Factory. Ilondoh et al. [19] used the direct conversion $\mathrm{X}$-ray TV camera [20] to measure directly the climb rate of dislocations, thereby yielding the self-diffusion coeflicient as a function of tempcrature.

Dynamic white radiation crystal growtl experiments have been undertaken by the Marseilles group of Gastaldi for over a decade (e.g. [21]). Recently a consortium led by Whitehouse has undertaken in silu MBE growth of InGaAs on GaAs at Daresbury Laboratory. A full MBE growth chamber capable of state-of-the-art epitaxy has been constructed with Be windows and can be mounted on the 6 circle 
diffractometer on station 9.4 of the SRS. Double axis X-ray topographs are taken in the Bragg geometry using asymmetric (grazing incidence) reflections. Presently these are recorded during growth interrupts on Nuclear Emulsions. The initial stages of the relaxation process have becil studied in detail for several In compositions on LEC, horizontal and vertical gradient freezc Bridgman (IIB, VGFB, respectively) grown substrates. We have shown that the misfit dislocation configurations seen at room tempcrature are representalive of those at the growth temperature and that during growth interrupts, the great majority of dislocations are stable. All initial misfit dislocations are nucleated ! y threading dislocations according to the mechanism of Matthews and Blakeslee (Fig. 1). A plateau in the

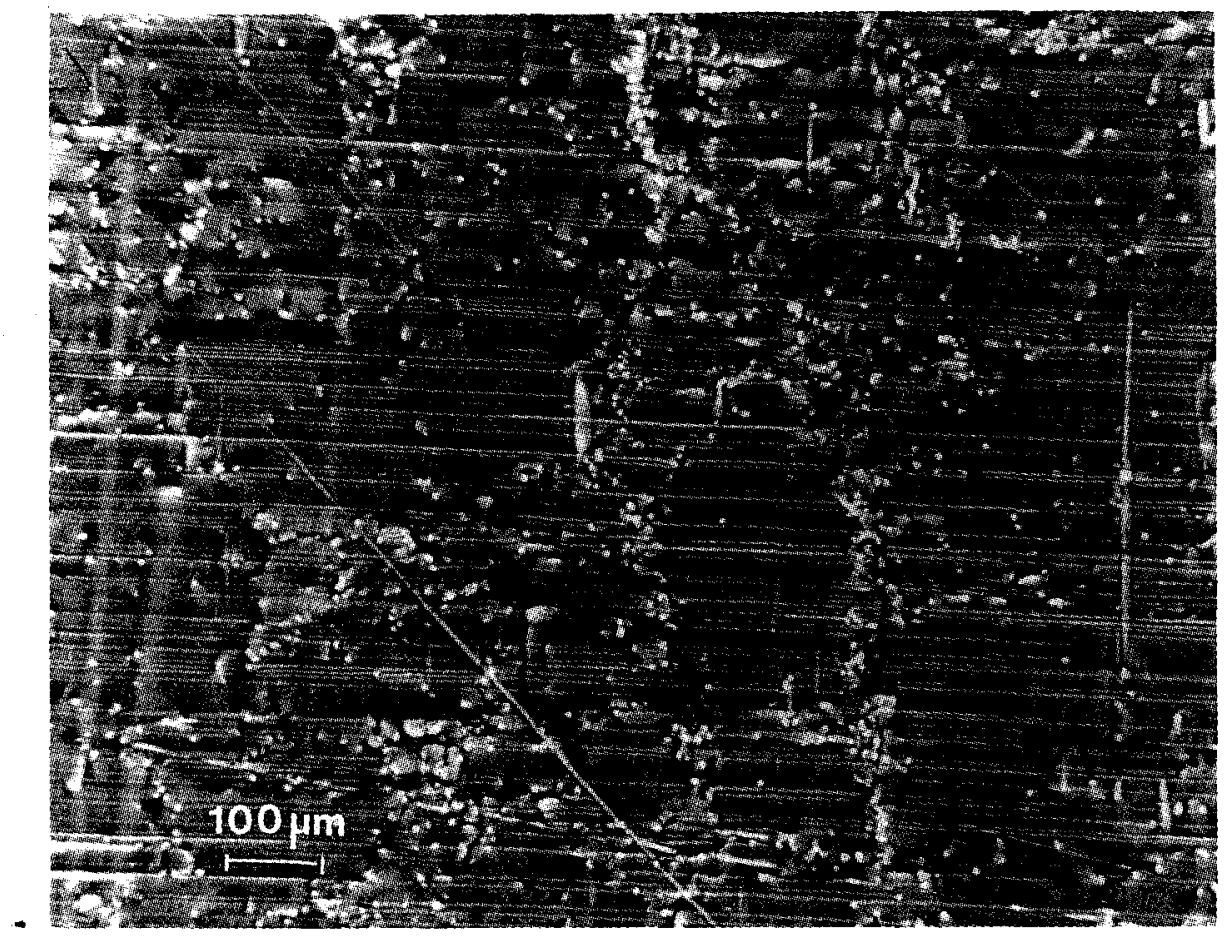

Fig. 1. Double crystal, Bragg-case synchrotron X-ray topograph of misfit dislocations at the $\mathrm{InGaAs} / \mathrm{Ga} \Lambda \mathrm{s}$ interface which are nucleatcd at threading dislocations.

relaxation occurs above the critical thickness for the nucleation of the very first misfit dislocations, corrcsponding to the elongation of misfit dislocations already nucleated [22]. In this region, for LEC and IIB substrates a strong asymmetry is observed in the initial relaxation [23]. Recent experiments on VGFB substrates have slown that this is associated with the distribution of threading dislocation Burgers vectors in the substrate. All misfit dislocations are of the fast glide type. The contrast of the misfit dislocations is strongly influenced by surface relaxation and full dynamical theory simulations [12] show that misfit dislocation images 
become very weak for very low epilayer thickness. Contrast is strongest for the highly asymmetric reflections chosen experimentally. Very rich structure is seen in the dislocation images when a thick GaAs capping layer is grown above the strained-layer.

\section{Stroboscopic topography}

The time structure of the synchrotron beam itself may be used in high frequency stroboscopy. The frequency may be as low as about $1 \mathrm{MIIz}$ in single-bunch mode and this was first utilised a decade ago in the study of travelling surface acoustic waves in SAW devices (intermediate frequency filters and delay lines), by Whatmore, Goddard, Tanner and Clark [24] and bulk wave devices by Gluer, Graeff and Moller [25]. Recently there has been a renewal of interest and, for example, Capelle, Zarka and co-workers have studied bulk waves in quartz resonators and used stroboscopy to identify unwanted modes associated with defects [26].

\section{Ultra-high sensitivity and triple axis topography}

White radiation topography suffers both from a high background and insensitivity to long range strains. Both problems are overcome by use of a beam conditioning crystal before the specimen. Compared with laboratory sources, there is a dramatic reduction in exposure time, which makes ultra-high sensitivity and high resolution triple axis topography feasible. Topographs of curved wafers show bands of diffracted intensity only where the Bragg condition is satisfied and for very low divergence beams, these bands can be very narrow. They can be used to plot the long range deformation in imperfect crystals by making multiple exposures on a single plate with the specimen moved by a constant angular step between exposures. One of the earliest synchrotron experiments was that of Ishikawa et al. [27] who performed plane wave topography with a separate monochromator-collimator in the $(++)$ setting to produce beautiful "zebra patterns" from a GaAs wafer containing a highly stressed region. Bilello et al. [28], working at Brookhaven, showed that high angular sensitivity, but low spatial resolution, $\mathrm{X}$-ray topography has far-reaching applications outside the confines of highly perfect crystals. They used a similar technique to measure the strains around cracks in fatigued zinc bicrystals. By use of several sets of Bragg contours in the double axis geometry, they were able to reconstruct the full tensor deformation field around cracks.

The importance of the semiconductor industry in Japan has naturally meant that very considerable effort has been expended in experiments to exploit the intensity and natural collimation of synchrotron radiation to increase the sensitivity to micro strains in extreme perfection silicon crystals. These experiments have all used one or more beam conditioning crystals to produce a pseudo-plane wave, a beam of angular divergence much less than the reflecting range of the specimen crystal. Such experiments are only practicable with extremely high intensity incident radiation. For example, double crystal experiments to study micro defects in silicon were performed by Chikaura and colleagues [29] and Iida et al. [30] have used extremely high order section topography with a grazing exit beam conditioner to study modified Czochralski silicon annealed at low temperatures. Kitano 
et al. [31] have used the triple axis topography method to examine the tilts and dilations around growth dislocations in bulk GaAs crystals. It is pleasing to note that Japanese scientists are using high sensitivity and resolution topography as a standard analytical tool at the Photon Factory. For example in the very precise measurements of the absolute lattice parameter of GaAs undertaken using the Bond method, Usuda et al. [32] used double axis topography to assess the relative perfections of different parts of the sample from which the lattice parameter measurements were made.

\section{Recent experiments at the European Synchrotron Radiation Facility}

Recent experiments by Baruchel et al. [33] at the ESRF using the Materials Science wiggler beamline have shown the potential of this new source for real time topography and the study of heavy elements. Pilot experiments showed exposure times, as predicted, in the millisecond regime. Due to the small source size, the spatial resolution remained good out to large specimen-to-detector distances, the variation being as calculated from the source and experiment geometry. Also, as expected, beam heating was a major problem in performing white radiation topography experiments of strongly absorbing materials, this being overcome by use of filters. The effect of heat load was not noticeable over the exposure time with metallic specimens. $A$ major success was the per[ormance at very short wavelengths. Excellent Kato fringes were observed at a wavelength of $0.21 \AA$ through a single crystal of silicon $1 \mathrm{~cm}$ thick. Similar success was obtained in the imaging of ferromagnetic domains in a Fe-3\%Si $1.4 \mathrm{~mm}$ thick. Until now, only neutron topography has been capable of imaging internal domain structures of such a thick material.

\section{References}

[1] A.R. Lang, Acta Crystallogr. 12, 249 (1959).

[2] B.K. Tanner, X-Ray Diffraclion Topograply, Pergamon, Oxford 1976.

[3] Characterisalion of Crystal Growth Defects by X-Rray Methods, Eds. B.K. Tanner, D.K. Bowen, Plenum, New York 1980.

[4] T. Tuomi, K. Naukkarinen, P. Rabe, Phys Slatus Solidi A 25, 93 (1974).

[5] M. IIart, J. Appl. Crysl. 8, 436 (1975).

[6] D.K. Bowen, B.K. Tanner, Maler. Sci. Rep. 8, 369 (1992).

[7] J.F. Petroff, M. Sauvage, P. Riglet, II. Hashizume, Philos. Mag. A 42, 319 (1980).

[8] T. Ishikawa, T. Kitano, J. Matsui, J. Appl. Crystallogr. 20, 344 (1987).

[9] T. Kitano, T. Ishikawa, J. Matsui, Plizlos. Mfag. A 63, 95 (1991).

[10] J. Miltat, in: Characterization of Crystal Growlh Defects by X-Ray Methods, Eds. B.K. Tanner, D.K. Bowen, Plenum, New York 1980, p. 401.

[11] W.J. Boettinger, R.C. Dobbyn, H.E. Burdette, M. Kuriyama, Nucl. Instrum. Methods 195, 355 (1982).

[12] W. Spirkl, B.K. Tanner, C. Whitehouse, S.J. Barnett, A.G. Cullis, A.D. Johnson, A. Keir, B. Usher, G.F. Clark, W. Hagston, C.R. Hogg, B. Lunn, Philos. Mag. A 69, 221 (1994); ibid (in press). 
[13] S. Ahmad, M. Ohtomo, R. Whitworth, Nature 319, 659 (1987).

[14] S. Ahmad, R. Whitworth, Philos. Mag. A 57, 749 (1988).

[15] C. Shearwood, R.W. Whitworth, J. Glaciology 35, 281 (1989).

[16] A. Higashi, T. Fukuda, K. Hondoh, K. Goto, S. Amaki, in: Dislocations in Solids, Eds. H.T. Suzuki, K. Ninomiya, K. Sumino, S. Takeuchi, University of Tokyo Press, Tokyo 1985, p. 511.

[17] C. Shearwood, R.W. Whitworth, Philos. Mag. A 64, 289 (1991).

[18] T. Hondoh, H. Iwamatsu, S. Mae, Plitos. Mag. A 62, 89 (1990).

[19] T. Hondoh, A. Goto, R. Hoshi, T. Ono, II. Anzai, R. Kawase, P. Pimienta, S. Mae, Rev. Sci. Instrum. 60, 2494 (1989).

[20] S. Suzuki, M. Ando, K. Hayakawa, O. Nittono, H. Hashizume, S. Kishino, K. Kohra, Nucl. Instrum. Methods 227, 584 (1984).

[21] J. Gastaldi, C. Jourdan, G. Grange, Philos. Mag. A 57, 971 (1988).

[22] C.R. Whitehouse, S.J. Barnett, A. Johnson, A.D. Pitt, A. Keir, G.F. Clark, W. Spirkl, B.K. Tamner, C.R. Hogg, B. Lunn, W. Hagston, in: Proc. Oxford Conf. on Microscopy of Semiconducting Materials, Ed. A.G. Cullis, I.O.P. Conf. Ser., Vol. 134, Bristol (U.K.) 1993, p. 563.

[23] G.S. Green, B.K. Tamner, S.J. Barnett, M. Emeny, A.D. Pitt, C.R. Whitehouse, G.F. Clark, Philos. Mag. Letts. 62, 131 (1990).

[24] R.W. Whatmore, P.A. Goddard, B.K. Tanner, G.F. Clark, Nature 299, 44 (1982).

[25] C.-C. Gluer, W. Graeff, H. Moller, Nucl. Instrum. Methods 208, 701 (1983).

[26] A. Zarka, B. Capelle, J. Detaint, J. Schwartzel, J. Appl. Crystallogr. 21, 967 (1988).

[27] T. Ishikawa, T. Kitano, J. Matsui, Jpn. J. Appl. Phys. 24, L968 (1985).

[28] J.C. Bilello, H.A. Sclımitz, D. Dew-IIughes, J. Appl. Phys. 65, 2282 (1989).

[29] Y. Chikaura, M. Imai, Jpn. J. Appl. Phys. 29, 221 (1990).

[30] S. Iida, H. Takeno, Y. Sugita, H. Kawata, Jpn. J. Appl. Phys. 29, 970 (1990).

[31] T. Kitano, T. Ishikawa, J. Matsui, Philos. Mag. A 63, 95 (1991).

[32] K. Usuda, S. Yasuami, Y. Higashi, H. Kawata, M. Ando, Jpn. J. Appl. Phys. 20, L210 (1990).

[33] J. Baruchel, J. Hartwig, F. Zontone, Synchrotron Radiation News 7, 19 (1994). 